\title{
Automated geodetic Very Long Baseline Interferometry observation and data analysis system
}

\author{
Yasuhiro Koyama ${ }^{1}$, Noriyuki Kurihara ${ }^{1}$, Tetsuro Kondo ${ }^{1}$, Mamoru Sekido ${ }^{1}$, Yukio Takahashi ${ }^{2}$, Hitoshi Kiuchi ${ }^{2}$, and Kosuke Heki $^{3}$ \\ ${ }^{1}$ Kashima Space Research Center, Communications Research Laboratory, Japan \\ ${ }^{2}$ Standards and Measurements Division, Communications Research Laboratory, Japan \\ ${ }^{3}$ National Astronomical Observatory, Japan
}

(Received December 24, 1997; Revised June 11, 1998; Accepted June 26, 1998)

\begin{abstract}
A precise geodetic measurement network using three modern space geodetic techniques, i.e. Very Long Baseline Interferometry, Satellite Laser Ranging, and Global Positioning System, is being established around Tokyo, Japan by the Communications Research Laboratory. The Key Stone Project, which is the name of the project, was started to obtain precise relative positions of four stations using these three space geodetic techniques on a daily basis. The system was designed to make frequent observations possible with minimum human operations and to provide analyzed results as fast as possible. This paper describes various aspects of new features and the performance of the automatic geodetic Very Long Baseline Interferometry observation and data analysis system designed for the Key Stone Project. This automated design has allowed daily Very Long Baseline Interferometry experiments to be conducted since January 1995 and the results to be immediately made available for public use after each experiment.
\end{abstract}

\section{Introduction}

Very Long Baseline Interferometry (VLBI), Satellite Laser Ranging (SLR), and Global Positioning System (GPS) are precise geodetic measurement methods and are often called space geodetic techniques (Anderson and Cazenave, 1986). Since these three techniques have different characteristics in many aspects, collocating them has been an idea of great interest in recent years. By placing different techniques close together, one can compare the different and independently obtained results with each other, improving their individual reliability. Communications Research Laboratory (CRL) has been conducting research and technical development of these three techniques and started a new research project to establish a compact space geodetic network using all three of these three space geodetic techniques in 1994. Observation facilities using them were located placed at four sites in the Kanto area of Japan, Koganei (Tokyo), Kashima (Ibaraki), Miura (Kanagawa) and Tateyama (Chiba) as shown in Fig. 1. The project was called the Key Stone Project after an ancient mythological story originated at Kashima. The relative positions of monuments at each site are precisely measured with local ground survey measurements. The network is relatively small considering that VLBI and other space geodetic techniques often use inter-continental baselines sometimes exceeding $10000 \mathrm{~km}$. But the smallness of the network can be regarded as an advantage since many technical challenges can be easily tested or applied. The network is unique in collocating three space geodetic techniques and it can be considered as a technical test-bed for precise geodetic research.

The developments of VLBI technique began in late 1960's

Copy right(C) The Society of Geomagnetism and Earth, Planetary and Space Sciences (SGEPSS); The Seismological Society of Japan; The Volcanological Society of Japan; The Geodetic Society of Japan; The Japanese Society for Planetary Sciences.
(Clark et al., 1985) and the technique has been extensively used in various fields of geodynamics such as global plate tectonic measurements (Ryan et al., 1993) and studies of variations in the Earth's rotation (Eubanks, 1993). Since the early 1980's, various technologies necessary for VLBI measurements have been developed also in CRL (Kunimori $e t$ al., 1993). Using the initially developed observation system, the 26- and 34-m antennas at Kashima Space Research Center (KSRC) of CRL have participated in global and regional VLBI sessions conducted by many organizations including CRL itself. Some of the international VLBI sessions conducted by the National Aeronautics and Space Administration (NASA) and all of the regional VLBI sessions conducted by CRL were processed by a VLBI correlator system developed at KSRC and the VLBI observations were analyzed to obtain valuable results. The experience and know-how learned while developing the observation and data processing systems for VLBI were utilized to develop the Key Stone Project VLBI system.

A major motivation of the Key Stone Project network was to perform precise space geodetic measurements as frequently as possible and to investigate the dynamic behavior of a relatively small region around Tokyo associated with seismic activities and deformation of the lithosphere in the area (Kurihara et al., 1996). The area around Tokyo is seismically active and shows a complicated distribution of deformation due to plate tectonic motions of three interacting plates. Continuous monitoring of the region is expected to provide valuable information about the geophysical processes occurring under the region. To achieve this objective, measurements have to be performed as frequently as possible and the results are required to be made available with minimum delay. Before the Key Stone Project, VLBI experiments took a long time to coordinate and to process the observed data. 


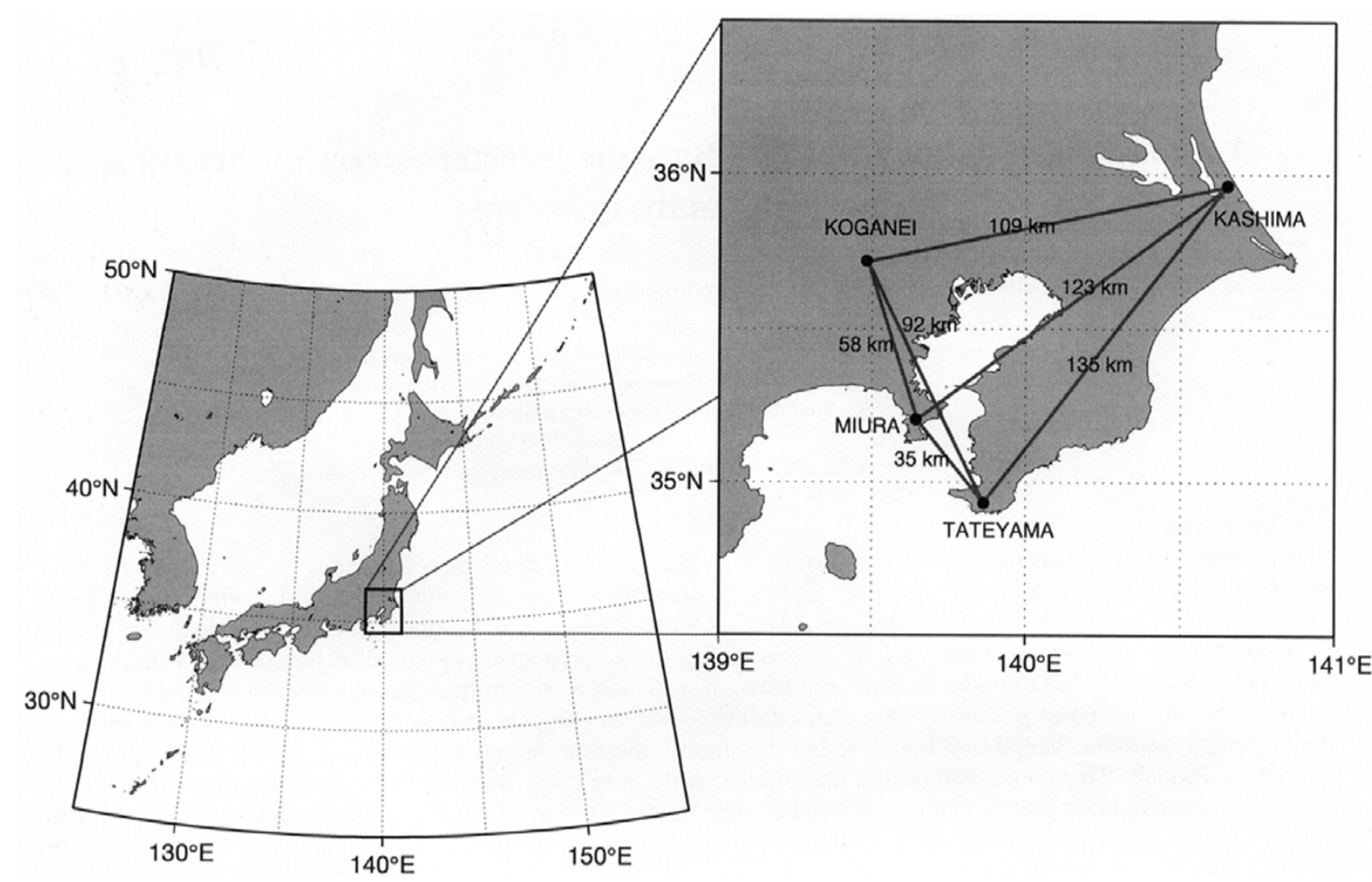

Fig. 1. Geographical locations of the four observation stations in the Key Stone Project space geodetic network. Baseline lengths range from $35 \mathrm{~km}$ to $135 \mathrm{~km}$.

In the case of Western Pacific VLBI Network experiments (Koyama et al., 1994), the total time required from preparation through data correlation and analysis was more than a month. The Key Stone Project VLBI observation and data processing systems were designed to achieve automated operation throughout the entire process. These systems have enabled frequent and regular VLBI observations to be made and reports of analyzed results to be published quickly. This paper mainly describes the observation and data analysis systems of the Key Stone Project VLBI network, focusing on various features providing automation and robustness.

\section{Observation Systems}

\subsection{Antenna and receiving systems}

Figure 2 is a picture of the $11-\mathrm{m}$ antenna system for VLBI observations at Kashima. The antenna systems at all four sites have identical designs except for the heights of the antenna pedestals. The antenna is a fully steerable Cassegrainian system equipped with S-band (2100-2500 $\mathrm{MHz}$ ) and X-band (7700-8600 MHz) receivers. Its pointing direction can be moved at a slewing rates of 3 degree per seconds for both azimuth and elevation angles. Table 1 shows the estimated System Equivalent Flux Densities (SEFDs) of the receiving systems. SEFD is the flux density of a hypothetical radio source that would generate equivalent power in the receiver output to the power of the system noise; it is often used to measure overall performance of a receiver system since this value is related with a signal-to-noise ratio

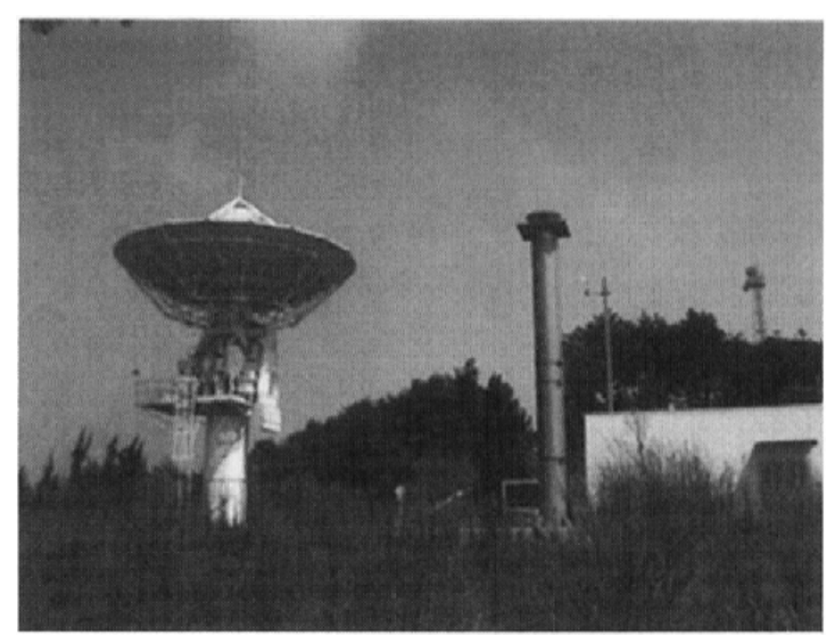

Fig. 2. The 11-m antenna system at Kashima. The GPS receiving antenna on the top of the steel pillar can also be seen in front of the VLBI antenna.

by an equation which will be discussed later. Although the basic designs of the receiving systems at the four stations are the same, their actual performances vary slightly. The SEFD values for the 34-m antenna at Kashima are also shown in the table for comparison. The temperature in the receiver cabinet located beneath the main reflector is maintained within 2 degrees of nominal to maintain phase stability of the signal path. A hydrogen maser system at each station is also lo- 
Table 1. System Equivalent Flux Density (SEFD) of four VLBI antennas. These values were estimated from a measurement of the 34-m antenna at Kashima and correlated flux results which were obtained in a joint VLBI experiment with the 34-m antenna station at Kashima and four Key Stone Project stations.

\begin{tabular}{lcc}
\hline Station & SEFD (X-band) & SEFD (S-band) \\
\hline Koganei & $6180 \mathrm{Jy}$ & $3030 \mathrm{Jy}$ \\
Kashima & $5490 \mathrm{Jy}$ & $2830 \mathrm{Jy}$ \\
Miura & $10220 \mathrm{Jy}$ & $3350 \mathrm{Jy}$ \\
Tateyama & $16490 \mathrm{Jy}$ & $3070 \mathrm{Jy}$ \\
\hline 34-m at Kashima & $316 \mathrm{Jy}$ & $343 \mathrm{Jy}$ \\
\hline
\end{tabular}

cated in a temperature controlled chamber to achieve better frequency stability.

The S-band signal is converted to the Intermediate-Frequency (IF) band between $500 \mathrm{MHz}$ and $900 \mathrm{MHz}$ using a local frequency signals of $3000 \mathrm{MHz}$. The X-band signal is split and converted using local frequency signals of $7200 \mathrm{MHz}$ and $7600 \mathrm{MHz}$ into two IF bands between 500 $\mathrm{MHz}$ and $1000 \mathrm{MHz}$, which correspond to 7700-8200 MHz and $8100-8600 \mathrm{MHz}$ of the original signal. Three IF band signals, one from the S-band and two from the X-band, are again converted using newly developed video converter units. Since the lower side band (LSB) of the local frequency signal is used for the IF band, the LSB outputs of the video converter units are used for the S-band observations.

\subsection{Data acquisition systems}

There are two main observation modes in the Key Stone Project VLBI system: a tape-based VLBI mode and a realtime VLBI mode. In the former, the radio signals received at each antenna are digitized and recorded on D-1 standard magnetic cassette tapes using a K-4 data recorder unit whose interface unit was designed for the Key Stone Project (Kiuchi et al., 1996a). The recorded tapes are transported after each experiment to Koganei station where a VLBI correlator system is located. In this case, observation data are correlated the day after an experiment and the final analysis results become available within two days after each experiment was performed. A minimum number of simple human operations is required at three remote stations to replace tapes and transport recorded tapes to Koganei station. These simple operations are performed by a local contractor at each site. The amount of effort required is, however, much less than for conventional geodetic VLBI experiments. Once the observation tapes have been set in the digital mass storage tape changing unit, the required ones are identified by bar-code labels and are loaded or unloaded to and from the data recorder unit automatically. At Koganei correlation center, additional human operations are required to organize the observation tapes. But these are also few in number and simple. The operations at the Koganei correlation center are to ship and receive observation tapes, place them in tape changers for observations and data correlation, and then start the VLBI data correlation processing. Correlation processing and data analysis are done without human interaction throughout. Figure 3



Fig. 3. VLBI observation racks in the observation control room adjacent to the 11-m antenna at Kashima. The rack on the left is for an antenna control unit and a video monitor system for the antenna. The rack in the middle is for controlling and monitoring the receiver. The right-hand rack is for analog unit of the backend system. The tape changing unit for the digital mass storage is located right of these three racks.

shows antenna control and data acquisition racks at Kashima station.

In the real-time VLBI mode, on the other hand, after the radio signals received at each antenna have been digitized and formatted by the data interface unit in the same manner as in the tape-based VLBI mode, the formatted data are transmitted to a real-time VLBI correlator located at Koganei station. Since the amount of data is huge, it is transferred by an optical fiber digital communication line using Asynchronous Transfer Mode (ATM). The four stations are connected by newly developed optical fibers that can transfer digital data at up to 2.4 gigabits per second (Gbps). The design of the correlator system used for the real-time VLBI mode has identical design with the correlator system used for the tape-based VLBI data processing. The ATM data transmitter and receiver interfaces are the key technical components developed for the real-time VLBI system of the Key Stone Project (Kiuchi et al., 1996b). In the case of real-time VLBI mode, no human operations are required at either the remote stations or the Koganei data correlation station. All the processes required during observations and data analysis are fully automated. The analyzed results become available immediately after the final observation of an experiment. Recent developments in the high speed data communication technology were remarkable. Maximum speed capability is glowing very fast and soon the real-time VLBI will become realistic for much longer baselines. The real-time VLBI technique has a potential to expand observation bandwidth and to improve the sensitivity which has been limited by the speed limitation of the data recording technology.

\subsection{Observation control and monitoring systems}

At each station, two workstations and one PC system are used to perform all the automated tasks necessary for VLBI observations, as shown in Fig. 4. The PC system, which is used to control tracking of the antenna system, is called antenna control PC. When it is given the right ascension and 




Fig. 4. Schematic diagram of the exchange of data between various equipment and the observation control workstation or the monitoring workstation.

the declination of a celestial radio source, the antenna control PC calculates its azimuth and elevation angles and sends necessary commands to the antenna control unit, which actually controls the motor servo systems of the 11-m antenna. The antenna control PC is connected to a Unix workstation called observation control workstation via a GPIB (General Purpose Interface Bus) communication interface. The observation control workstation provides right ascensions and declinations of target radio sources during a VLBI experiment according to an observation schedule file. Tape changing and data recording are also controlled by the observation control workstation in the tape-based VLBI mode of operations. The observation schedule file also specifies local frequencies and video-bandwidth of 16 observation channels, and necessary commands are sent from the observation control workstation to the data acquisition terminal via the GPIB communication bus to set up observation configurations at the beginning of each experimental session.

In a geodetic VLBI experiment, weather data and cable delay data have to be collected during the experiment for the calibrations required in the data analysis. These data are collected by a different Unix workstation called the monitoring workstation. This workstation also measures and collects many data for monitoring the statuses of many components via a GPIB interface and a parallel I/O interface. These tasks are separated from the observation control workstation so that calibration data as well as monitoring data will be collected continuously regardless of whether the VLBI observations are being performed or not. Separating data monitoring tasks from observation control tasks also reduces the data load on the GPIB line. When one of the status signals collected by a parallel I/O interface indicates a problem, or when one of the monitored data items exceeds a predetermined threshold, the monitoring workstation indicates the problem on the console terminal screen and sends an alarm signal to the central control workstations over the wide area network system.

\subsection{Central control systems}

At Koganei and Kashima stations, there are additional Unix workstations called central control workstations. Their role is to organize observations performed by the observation control workstations at the four stations and to collect status and monitoring data from the monitoring workstations. The graphical user interface of the central control workstations has been designed to be easy to use so that untrained operators can easily perform remote operations and various checks on the observation systems at remote sites. Observations at remote sites can be started, interrupted, and resumed after an interruption from the central control workstations. Receiving systems and data acquisition systems can be controlled remotely, and the results of the operations can be confirmed with video camera images over the computer network. Any problems detected by an observation control workstation or a monitoring workstation are reported to the central control workstations and such events are logged in a file and sent over the network to pre-registered e-mail addresses to notify the designated persons in charge that there is a problem. Observation programs on the observation control workstations are executed by commands from two central control workstations. These two commands are issued at different times with a certain arbitrarily-chosen interval between them. In 


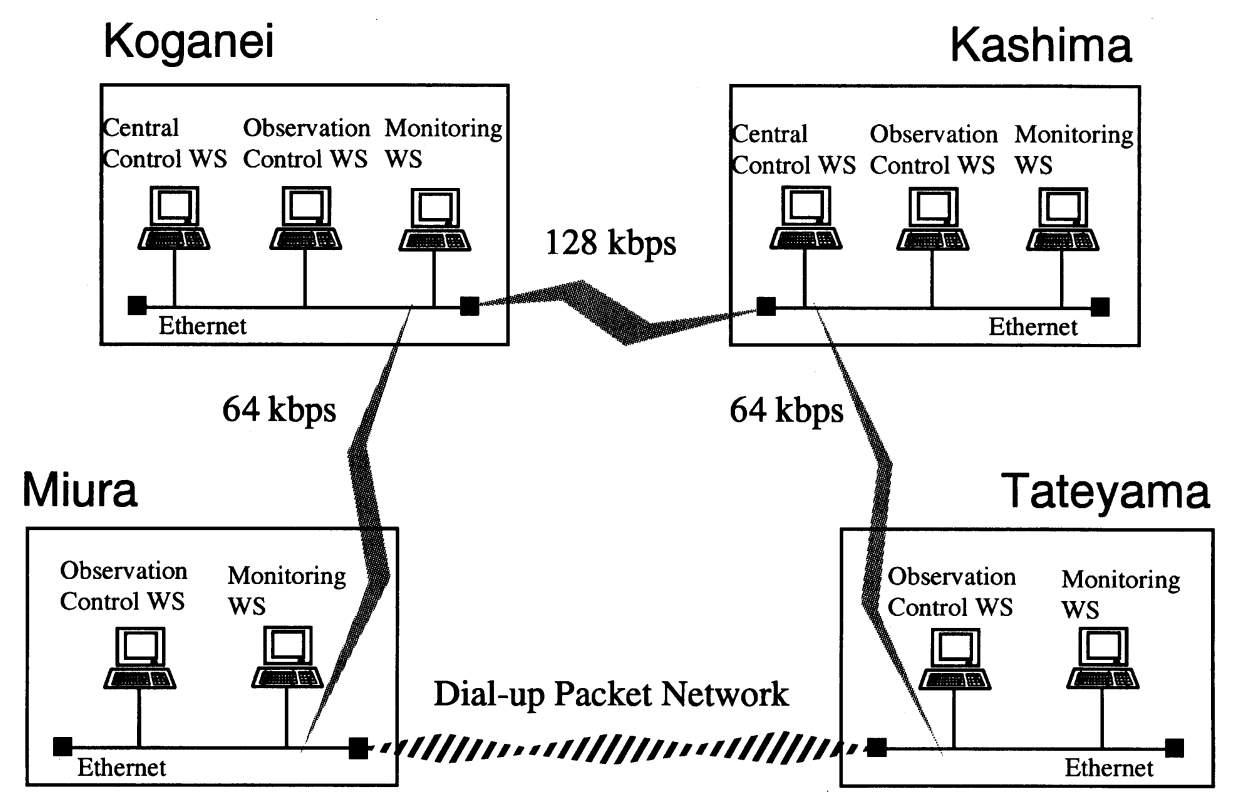

Fig. 5. Configurations of the wide area computer network system for the entire network of the Key Stone Project VLBI network. The dial-up network line between Miura and Tateyama stations becomes active when one of the other three networks happens to be unavailable. The high-speed (2.4 Gbps) ATM network lines used to transfer observed data in the real-time VLBI mode are not used for the communications between computer systems and are not shown here.

the usual case, time which can be arbitrary set. In an usual case, the first command starts the observations at all four sites, and the second command is ignored. However, if the first command fails to reach the observation control workstation at any station for some reason, then the second command becomes effective and prevents the experiment from failing as a result of an unexpected situation.

After all the observations in an experiment have been done, observation $\log$ files are transferred and merged with other data files obtained by monitoring workstation at each site. The merged log files are then used in the data correlation processing and when the database files are created. Before the experiment, the central control workstations perform check routines at all four stations to examine whether the observation tapes are ready. If any of the observation tapes are not properly set in the tape changer unit or have a problem, an e-mail message is sent to the responsible persons to inform them that the station is not ready for the observations. The tapes to be used in the experiment are specified in a master control file stored on each central control workstation. The central control workstations interpret the master control file to determine which stations will participate in the next experiment and which tapes should be used at each station. The master control file is updated every month automatically so that regular experiments can be done without human interaction. The master control file can also be modified at any time by an operator on a central control workstation in order to change the participating stations and tapes to be used. Every time the file is modified, the master control file is sent to the other central control workstation to ensure consistency between these files on the two central control workstations.

\subsection{Computer network system}

At each station, a TCP/IP local area network over Ethernet connects the observation control workstation and the moni- toring workstation; at Koganei and Kashima stations, it also connects the central control workstation. Other computers are connected to the same network to acquire data from a GPS receiver and a seismometer. In addition, at Koganei station, correlator units and additional workstations required for the correlation processing and data analysis are connected to the network. An intelligent network bridge is located between the segment for correlators and the segments for other computers to keep the huge amount of correlated data on one side.

All four VLBI stations are connected together by dedicated computer network lines. The configuration of the network system is shown in Fig. 5. Three dedicated computer network lines connect Koganei to Miura, Koganei to Kashima, and Kashima to Tateyama. Since the data traffic on the computer network is not very high and there are no requirements for high-speed responses between the computer systems, $64 \mathrm{kbps}$ is sufficient as the data capacity of the network system. However, the data rate between Kashima and Koganei is doubled to $128 \mathrm{kbps}$ because most of the communications are done by two central control WS systems at Kashima and Koganei with other WS systems and therefore the data traffic on this segment is about twice as large as the other two segments. In addition to three dedicated computer network lines, Miura and Tateyema stations are connected by a dial-up packet line, which becomes active if one of the three dedicated lines becomes unavailable. This configuration ensures that each station is always reachable from any other station, so observations can be performed as usual unless two or more network lines fail at the same time.

The network is connected to the Internet through the data analysis workstation at Koganei. This workstation has two network interfaces: one is connected to the Key Stone Project network and the other is connected to the laboratory network, 
which is connected to the public Internet. This workstation acts as a firewall system. Necessary connections between two network segments can be made through the workstation, but unauthorized access to the Key Stone Project network from the public Internet is prevented.

The antenna control PC and observation control workstation at each station have parallel data interfaces, which are connected to an Inter-Range Instrument Group (IRIG) timecode signal generator of a GPS receiver. The internal clock of these computer systems are synchronized to the coordinated universal time (UTC) provided by the GPS receiver every day during the observation preparation procedures. The precise time information maintained in the antenna control PC is necessary to point the antenna to the direction of a radio source accurately. The observation control workstation disseminates precise time information by Network Time Protocol (NTP) as a server. The monitoring workstations and central control workstations are configured as NTP clients and the internal clocks of these systems are always synchronized to the UTC.

\subsection{Redundant system design concepts}

Since the VLBI observation system of the Key Stone Project was intended to achieve automated operation as much as possible, it was designed with a redundant configuration to improve overall reliability. The network system described in the previous section is one example which has been designed with the redundant concept. Each observation control workstation is also accessible via a public telephone line using a modem, and observations can be controlled remotely even if the network connection to the station is unavailable.

The two central control workstation systems at Koganei and Kashima are identical and operate independently from each other. This dual operation guarantees that regular observations can be made at all stations even if one of the central control workstations malfunctions. The combination of redundant designs makes the entire VLBI observation system robust against many possible irregularities. If any one station goes down for any reason, the other three stations can still continue observations because they are accessible from at least one of the central control workstations via the dial-up packet network line. Some of the software components related to automated experiment management are also written with redundant design. For example, the observation schedule file is created two weeks before each experiment. This file contains all the information necessary to carry observations and correlation processing of an experiment. Especially, the sequence of radio sources to be observed can not be the same and the schedule file have to be made every time. But whether the file was actually created or not is checked again on the day of the experiment. If the file was not found, it is created at this time. In this way, the schedule file can be replaced anytime during the two weeks before the day of the experiment if a special experiment have to be coordinated, and on the other hand, the risk of the loss of the experiment because the schedule file was not created can be reduced considerably. This kind of redundancy is quite effective to improve the reliability of the automated observation system.

\section{Observations}

\subsection{Optimized observation time}

The Key Stone Project VLBI system uses relatively small and fast slewing antennas. Each antenna is equipped with low-noise amplifiers for the X- and S-band frequencies that operate without a cryogenic system. Using non-cryogenic receivers reduces the amount of maintenance and the likelihood of system failure but increases the system noise temperature compared with using cryogenic ones. Both the small antenna aperture and the high system noise temperature contribute to reduce the sensitivity of the receiving system, so only strong radio sources had to be selected for the Key Stone Project VLBI experiments. To obtain sufficient signal-to-noise ratio (SNR) $R$ within a reasonable correlation integration time $T$, the necessary flux density $F_{\mathrm{s}}(\mathrm{Jy})$ of an observation target radio source after a cross correlation can be determined from the following relationship.

$$
R<\frac{2}{\pi} \cdot \frac{F_{\mathrm{s}}}{\sqrt{F_{1} F_{2}}} \sqrt{2 B T}
$$

where $B$ is the total frequency width $(\mathrm{Hz})$, and $F_{i}(i=1,2)$ are SEFDs (Jy) of the receiver systems at two stations. The factor $2 / \pi$ is a result of a loss due to the 1-bit sampling digitization error. If we limit the maximum correlation integration time to 320 seconds and minimum SNR to 10 , then the minimum flux density of the radio sources with an observation data rate of $64 \mathrm{Mbps}$ is $1.8 \mathrm{Jy}$ in the X-band and $0.57 \mathrm{Jy}$ in the S-band. From the list of radio sources whose positions are known precisely in the International Celestial Reference



Fig. 6. Data synchronization time for different observation modes and different observation data rates. 
Table 2. Optimized observation times (seconds).

\begin{tabular}{|c|c|c|c|c|}
\hline \multirow[t]{2}{*}{ Radio sources } & \multicolumn{2}{|c|}{ Real-time VLBI mode } & \multicolumn{2}{|c|}{ Tape-based VLBI mode } \\
\hline & $256 \mathrm{Mbps}$ & $64 \mathrm{Mbps}$ & $256 \mathrm{Mbps}$ & $64 \mathrm{Mbps}$ \\
\hline $0059+581$ & 332 & 332 & 360 & 360 \\
\hline $3 \mathrm{C} 84$ & 62 & 62 & 90 & 90 \\
\hline $0420-014$ & 117 & 222 & 145 & 250 \\
\hline $0552+398$ & 62 & 67 & 90 & 95 \\
\hline $0727-115$ & 126 & 239 & 154 & 267 \\
\hline $4 C 39.25$ & 62 & 62 & 90 & 90 \\
\hline $3 \mathrm{C} 273 \mathrm{~B}$ & 62 & 62 & 90 & 90 \\
\hline $3 \mathrm{C} 279$ & 62 & 62 & 90 & 90 \\
\hline $1308+326$ & 108 & 204 & 136 & 232 \\
\hline $1334-127$ & 62 & 98 & 90 & 126 \\
\hline $3 \mathrm{C} 345$ & 62 & 62 & 90 & 90 \\
\hline NRAO530 & 62 & 62 & 90 & 90 \\
\hline $1921-293$ & 62 & 62 & 90 & 90 \\
\hline $2134+004$ & 62 & 62 & 90 & 90 \\
\hline $2145+067$ & 62 & 94 & 90 & 122 \\
\hline $3 \mathrm{C} 454.3$ & 62 & 62 & 90 & 90 \\
\hline
\end{tabular}

Frame, 16 strong radio sources that satisfy the requirements were selected. Then the optimal integration time of crosscorrelation processing for each source was determined to get a sufficient SNR of 20 in both the X- and S-bands for all baselines. The maximum correlation integration time is limited to 320 seconds even if the desired SNR is not obtained and the minimum correlation integration time of 50 seconds is kept.

To determine an adequate observation time for the target radio sources, a certain amount of time required to synchronize data in correlation processing should be added to the optimized integration time. Figure 6 shows the distribution of the data synchronization times for two data rates and two observation modes. It reveals a clear difference in the data synchronization times between tape-based and realtime VLBI modes. In the real-time VLBI mode, the data synchronization time is about 10 seconds for data rates of both 256 and $64 \mathrm{Mbps}$, and more than $95 \%$ of the data were less than 12 seconds. On the other hand, in the tape-based VLBI mode, the data synchronization is achieved by adjusting the data reproducing timing mechanically, which takes longer than the data synchronization using data buffers, as is done in the real-time VLBI mode. The distribution of time required to perform data synchronization in this case has a peak at about 30 seconds, and it takes 40 seconds to cover $95 \%$ of all the data. The observation time for each radio source was determined by adding the correlation integration time determined from the SNR requirements and the data synchronization time, which is 12 seconds for the real-time VLBI mode and 40 seconds for the tape-based VLBI mode.
The results are shown in the Table 2. The maximum number of observations in one experiment is achieved at a data rate of $256 \mathrm{Mbps}$ in real-time VLBI mode. In a typical experiment with 24 hours of duration, about 600 observations are possible at $256 \mathrm{Mbps}$ in real-time VLBI mode, compared to only about 380 at $64 \mathrm{Mbps}$ in tape-based VLBI mode. The uncertainties of the estimated site coordinates are proportional to the inverse of the square-root of the number of observations if the signal-to-noise ratio is kept and each measurement can be regarded as independent from other observations. Therefore the final results are expected to be improved by about $26 \%$ by making observations at $256 \mathrm{Mbps}$ in real-time VLBI mode compared with ones at $64 \mathrm{Mbps}$ in tape-based VLBI mode. However the actual improvements were not so remarkable probably because the measurement errors can not be regarded as a white noise.

\subsection{Optimized channel frequency assignments}

The Key Stone Project VLBI observation system can perform observations with 16 video channels. Ten channels are assigned for the X-band and the other six for the S-band. The frequency assignments of observation channels were determined to achieve a wide equivalent frequency width after bandwidth synthesis processing while the side-lobe peak height of the bandwidth synthesis function was kept small. Table 3 shows the frequency assignments used at present. The phase calibration signal is injected into the received signal at $5 \mathrm{MHz}$ frequency intervals, and the local frequency of each channel is chosen to have the phase calibration signal at $10 \mathrm{kHz}$. 
Table 3. Frequency assignments.

\begin{tabular}{ccccc}
\hline \multicolumn{2}{c}{ X-band } & & \multicolumn{2}{c}{ S-band } \\
\cline { 1 - 2 } \cline { 5 - 5 } Ch. No. & Local Freq. (MHz) & & Ch. No. & Local Freq. (MHz) \\
\hline 1 & 7714.99 & & 11 & 2154.99 \\
2 & 7724.99 & & 12 & 2164.99 \\
3 & 7754.99 & & 13 & 2234.99 \\
4 & 7814.99 & & 14 & 2294.99 \\
5 & 8034.99 & & 15 & 2384.99 \\
6 & 8234.99 & & 16 & 2414.99 \\
7 & 8414.99 & & & \\
8 & 8524.99 & & & \\
9 & 8564.99 & & & \\
10 & 8584.99 & & & \\
\hline
\end{tabular}

\subsection{Automated VLBI observations}

In both tape-based and real-time VLBI experiments, the observations at four stations are performed without any operators. First, the observation schedule file is created by the software SKED using its AutoSKED capability. This software has been developed by NASA Goddard Space Flight Center and can produce an optimized observation schedule automatically (Steufmehl, 1983). The software is run by a clock daemon (cron) process of a Unix workstation two weeks before the actual observations. The observation schedule file is duplicated and transferred to two central control workstations. For better reliability, the observation schedule for the day is checked by a different cron process. If the schedule file was not actually prepared two weeks ahead, the schedule file is newly produced at this time. The observation mode and starting and ending times are specified in a parameter file and the observation schedule is produced according to its contents. These parameters can be easily modified using one of the widely available browsers for the World Wide Web (WWW). If a special observation schedule is required temporarily, an operator at Koganei central station can create the schedule file using a WWW browser.

On the day of the experiment, the two central control workstations independently transfer the observation schedule file to the four observation control workstations. Stations to be included in the session are controlled by the master control files on the central control workstations. Then the observation control program on the observation control workstation is started by a command from the central control workstation. Before the first observation is begun, the internal clock of the observation control workstation is synchronized to UTC using the time code generator of a GPS receiver, and then the input interface units of the data acquisition system are synchronized to the internal clock of the observation control workstation. This step is especially important when a leap second is introduced. The frequency settings of the video converter units and the observation data rate of the data acquisition system are properly set according to the informa- tion in the observation schedule file. During an experiment, the status of each site is reported to an operator at Koganei central station by e-mail once every hour. After all observations in the experiment have finished, the log files are transferred to the central control workstations and merged with the weather calibration data file, delay calibration data file, and time difference data files for the correlation processing and the following data analysis procedures.

\subsection{Regular observations}

The first VLBI experiment with the Key Stone Project network was performed for 24 hours on August 29, 1994 with the single baseline between Koganei and Kashima. Daily VLBI experiments began on January 31, 1995 with the same baseline. In the daily experiments, observations were usually performed for 5.5 hours from midnight. Full network experiments with the four stations shown in Fig. 1 started on September 1, 1996 on a daily basis. Table 4 shows how the reliability of the observation system of the Key Stone Project VLBI network has improved since full network experiments began in September 1996. The ratio of the number of successful experiments to the number of days in the month is shown in the table for each station. Here, an experiment was considered to be successful for a station if the station position could be estimated from the obtained data. As shown in the table, the ratio gradually improved, and all the experiments were successful for every station in February 1997. This demonstrates the reliability of the observation system designed for the Key Stone Project VLBI system.

Until September 29, 1997, daily VLBI observations were performed from 15:00 UT to 20:30 UT. From September 30,1997 , the frequency of experiments was reduced from every day to alternate days and the duration of an experiment was extended to 23.5 hours in the expectation that this would reduce systematic errors in the data analysis results (Kondo et al., 1998).

\section{Data Reduction and Analysis Systems \\ 4.1 Cross correlation processing}

The cross correlation processing of the observed data is done by the VLBI correlators located at Koganei station. There are two sets of cross correlation processing units: one is used for the tape-based VLBI mode and the other for the real-time VLBI mode. No human operations are required in the real-time VLBI mode. The correlation management program is always running, and the cross correlation processing is performed whenever the observations are performed (Kondo et al., 1996). In the tape-based VLBI mode, on the other hand, a human operator must set the data tapes in the tape changing unit of the correlator system and start the correlation management program. Once the cross correlation processing has begun, all procedures are performed automatically until the database files are created from the observation data.

\subsection{Initial data analysis}

In both tape-based and real-time VLBI modes, the output data from the correlator system at Koganei station are processed by the bandwidth synthesis program to determine time delays for all combinations from participating stations. The set of obtained time-delays and their rates of change is then combined with auxiliary data including meteorological 
Table 4. Ratio (\%) of successful experiments to the total number of days in a month summarized for every month from September 1996. An experiment was considered successful for a specific station if the station coordinate was estimated or used as a reference in the data analysis.

\begin{tabular}{clcccc}
\hline \multirow{2}{*}{1996} & Koganei & Kashima & Miura & Tateyama \\
\cline { 3 - 5 } & September & 96.7 & 96.7 & 83.3 & 93.3 \\
& October & 96.8 & 93.5 & 96.8 & 93.5 \\
& November & 93.3 & 80.0 & 93.3 & 83.3 \\
& December & 96.8 & 90.3 & 100.0 & 100.0 \\
& January & 90.3 & 96.8 & 100.0 & 100.0 \\
& February & 100.0 & 100.0 & 100.0 & 100.0 \\
& March & 90.9 & 100.0 & 100.0 & 86.4 \\
\hline
\end{tabular}

data and delay calibration measurements and is stored in two database files, one for X-band data and the other for S-band data. Only the X-band database is used for data analysis and the S-band database is mainly used to obtain correlated amplitudes of observed radio sources. The databases are created using Mark-III database handler software developed at NASA Goddard Space Flight Center and the file format of the databases is based on the Mark-III database file format, which can be handled by various software programs based on the Mark-III database handler routines.

Once the X-band database file has been created on the data analysis workstation at Koganei station, a set of data analysis programs processes the database file until the initial analysis results are obtained. Figure 7 illustrates the entire process of data analysis. At first, the DBUPDATE routine updates the database with the latest a priori information. The a priori information to be updated includes coordinates ( $\delta x$ and $\delta y)$ of the Earth's rotation pole with respect to the conventional pole, UT1-UTC, nutation offsets from the IAU80 standard model, terrestrial coordinates of reference points of VLBI stations, and coefficients for site displacements due to ocean loading effects. The same routine is used in the re-analysis procedures when a part of the a priori information is improved. In the next step, the CALC routine calculates theoretical delays and delay rates based on the a priori information and calculates a Jacobian matrix with various parameters for the least squares estimates. After the CALC routine, the database is ready for the least squares analysis. DATSET then extracts necessary information from the database and stores it in a work file. After these procedures, the VLBEST routine runs three or more times and least squares analyses are performed using different combinations of estimation parameters depending on the stage of the analysis. In the first step, only the clock offsets and their rates of change with respect to a reference station are estimated and the other parameters are fixed. In this procedure, the observed delays may include delay ambiguities, which are certain amounts of time multiplied by integers. The ambiguities are the results of the bandwidth synthesis processing, and the minimum step of ambiguity is defined by the inverse of the greatest common divisor of frequency spacings of the local frequencies of observation channels, which is 100 nanoseconds for both the X- and S-band frequency assignments in Table 3. The ambiguities are resolved using the residuals of delays after the least squares adjustment. In the second step, clock offsets at a time interval of 1 hour and site coordinates of all stations except for a reference station are estimated by the routine VLBEST. Observed delays having large residuals are flagged and removed from the following analysis by the routine MRKOBS. If the residual is greater than three times the root mean squared (rms) of the residual delays and also greater than 200 picoseconds (ps), it is regarded as a bad datum. This step is iterated until there are no more actual data to be removed. In the last step, the wet tropospheric zenith delays at a time interval of 3 hours are added to the parameters to be estimated, and the final results are obtained. The quality of the results are evaluated by the final $\mathrm{rms}$ of the delays. If the rms residual is smaller than a certain criterion (e.g. $100 \mathrm{ps}$ ), the results are regarded as reliable and are made accessible via WWW and anonymous ftp (file transfer protocol) over the Internet. If the residual $\mathrm{rms}$ exceeds the criterion, an alarm message is sent to pre-registered e-mail addresses. In this case, the database is analyzed manually and the results are finally publicized if reliable ones are obtained.

\subsection{Updating data analysis results}

In addition to the normal data analysis procedure, results are updated when any of the a priori information is improved. The most frequent case of this is when the International Earth Rotation Service (IERS) issues a bulletin. IERS is responsible for providing up-to-date series of parameters related with Earth's rotation and issues Bulletin-A twice a week and Bulletin-B once a month. At the time of initial data analysis, only the predicted values of $\delta x, \delta y$ and UT1-UTC are available as a priori information. No predictions are provided for the nutation offsets, so these values should be obtained using empirical model. The values of these parameters are improved in the bulletins issued after the experiment. The bulletins are received by e-mail and are processed automatically by the data analysis software. All the databases for which a priori information is improved are updated with the improved a priori information by the routine DBUPDATE. These databases are then processed by the routine CALC, and finally the parameters are reanalyzed by the routines DATSET and VLBEST.

The uncertainties of the predicted Earth Rotation Param- 


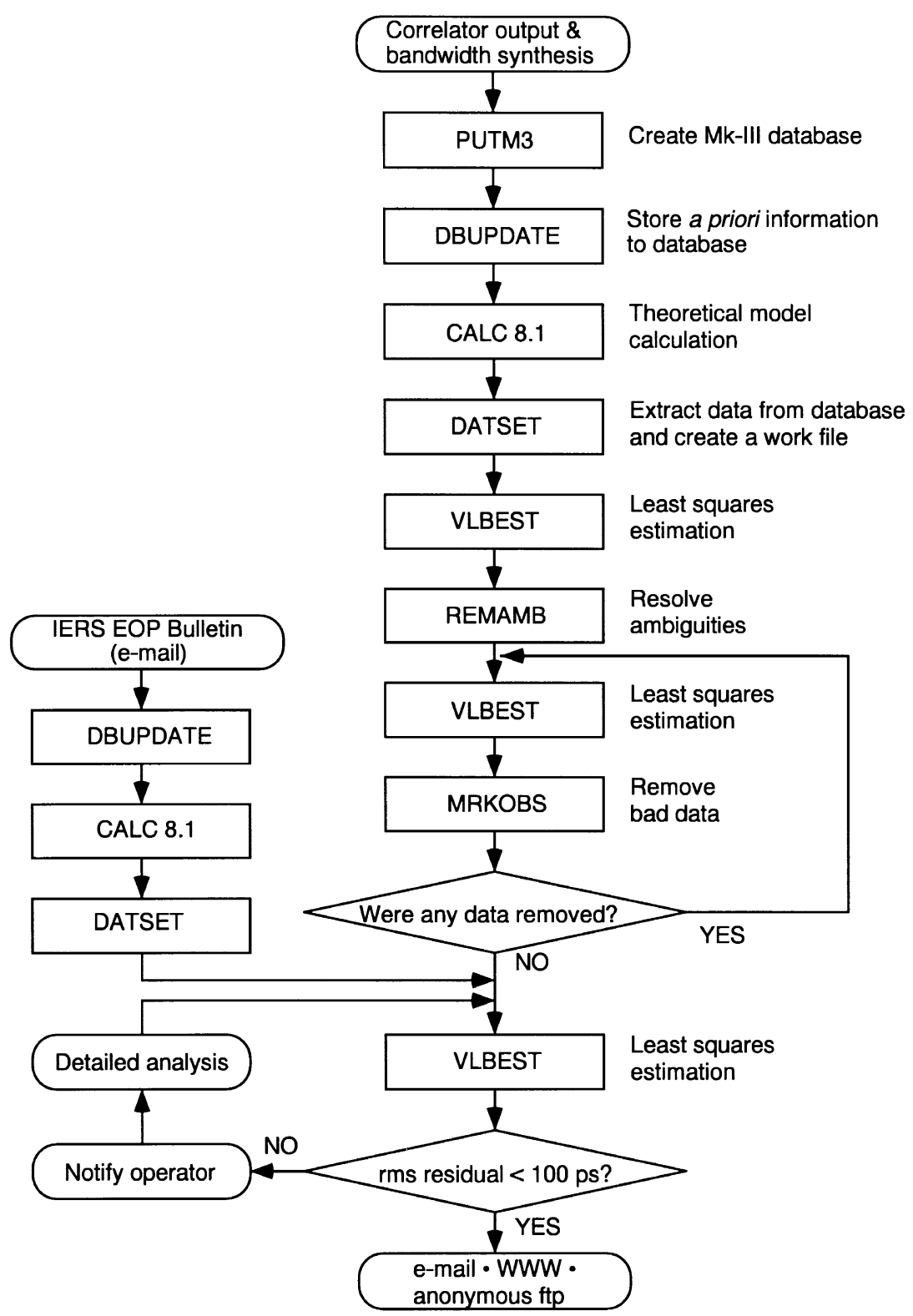

Fig. 7. Schematic data flow and block diagram of the automated VLBI data analysis.

eters are estimated as 3.8 mas (mas: milli arc seconds) for $\delta x$ and $\delta y$, and $1.5 \mathrm{~ms}$ for UT1-UTC for a prediction time of 10 days (IERS, 1995). In Fig. 8, the actual offsets of the three Earth Rotation Parameters published in the IERS Bulletin-A issued from January 1996 through August 1997 are compared with the final values that appeared in the IERS Bulletin-B. The actual prediction errors in the IERS Bulletin-A were worse than the values published in the IERS documents. From the comparison, the rms prediction variations of the $\delta x$ and $\delta y$ were evaluated as 5.1 mas $(\delta x)$ and 4.3 mas $(\delta y)$ at a prediction time of 10 days, and 3.1 mas $(\delta x)$ and 2.7 mas $(\delta y)$ at a prediction time of five days, whereas the rms prediction variations of the UT1-UTC were evaluated as 2.4 and $1.2 \mathrm{~ms}$ at a prediction time of 10 and 5 days, respectively. In the Key Stone Project VLBI initial data analysis, the predicted values of the Earth Rotation Parameters in the latest IERS Bulletin$A$ are used and the prediction time increases to 5 days in the worst case. This means that the three-dimensional site positions estimated from the initial data analysis are affected by the prediction errors. The 4.3 mas of the Earth's rotation pole position errors and the $1.2 \mathrm{~ms}$ of UT1-UTC error correspond to site position estimation errors of 2.4 and $9.6 \mathrm{~mm}$ in the horizontal and vertical components, respectively, for a baseline $110 \mathrm{~km}$, which is roughly the distance between Kashima and Koganei stations. The discussions above are based on the rms prediction variations of the Earth Rotation Parameters, so the actual site position errors may sometimes be larger.

Therefore, the data analysis should be repeated when the new IERS bulletin arrives and more accurate Earth Rotation Parameters become available. When a new IERS bulletin arrives by e-mail, all the databases containing Earth Rotation Parameters for which new values are now available are reanalyzed by the automated data analysis system. The errors 




Fig. 8. Errors in predicting the positions ( $\delta x$ and $\delta y)$ of the Earth's rotation pole and UT1-UTC in the weekly IERS bulletins compared with the final values in the monthly IERS bulletins.

in the parameters before the bulletin issue date are small and the site position errors due to them can be ignored.

\subsection{Results of experiments}

Figure 9 plots the estimated lengths of the baseline between Koganei and Kashima stations. Figure 10 shows the three dimensional site position of the Koganei station. The formal error of each estimation is shown by a vertical bar in both figures. The variations in baseline length and three components of site position are fitted by linear lines and the best fit lines are shown by straight solid lines. The reduced chisquared value of the fit for the baseline length data in Fig. 9 is 3.0 and the weighted rms of residuals is $3.1 \mathrm{~mm}$. The values for the other baselines and horizontal components of site positions are similar whereas the weighted rms of residuals for vertical components of site positions are larger $(17.6 \mathrm{~mm}$ for Koganei station for example). The fact that the reduced chi-squared value is significantly greater than 1.0 indicates that either all the formal errors were underestimated or there may have been a systematic error with seasonal variation. In the figure, one can also see evidence of gradual improvements in data quality with time. The results after September 30, 1997 are remarkable, reflecting the extended duration of each experiment. The formal errors in baseline length estimates decreased to about $1 \mathrm{~mm}$ and, if only the data after September 30, 1997 were used, the weighted rms residual would be about $2 \mathrm{~mm}$. Estimated three-dimensional site po- 


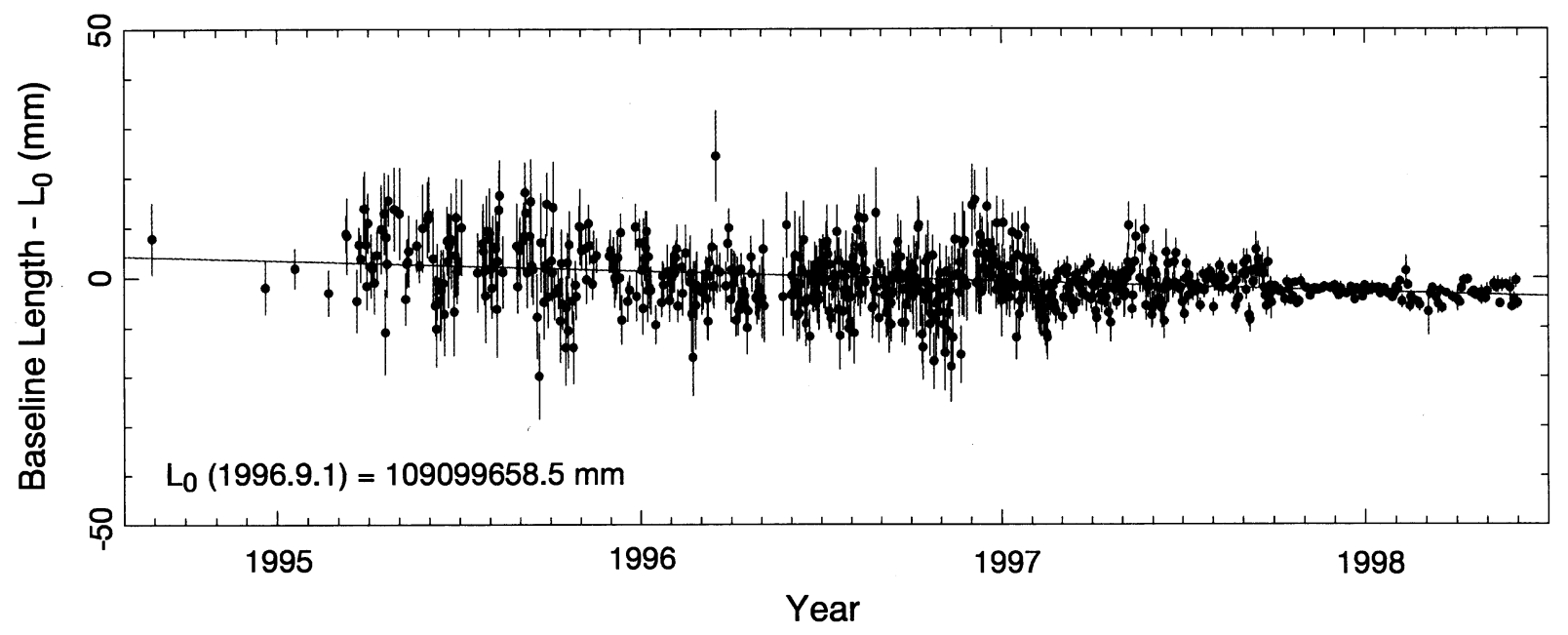

Fig. 9. Length of the baseline between Kashima and Koganei stations estimated from the Key Stone Project VLBI experiments. Estimated uncertainties in the estimates are shown by the one-sigma formal error bars. The epoch value of the baseline length $L_{0}$ and the rate of change were estimated from the entire data set by the least squares estimation and $L_{0}$ was subtracted from each data points in the figure.
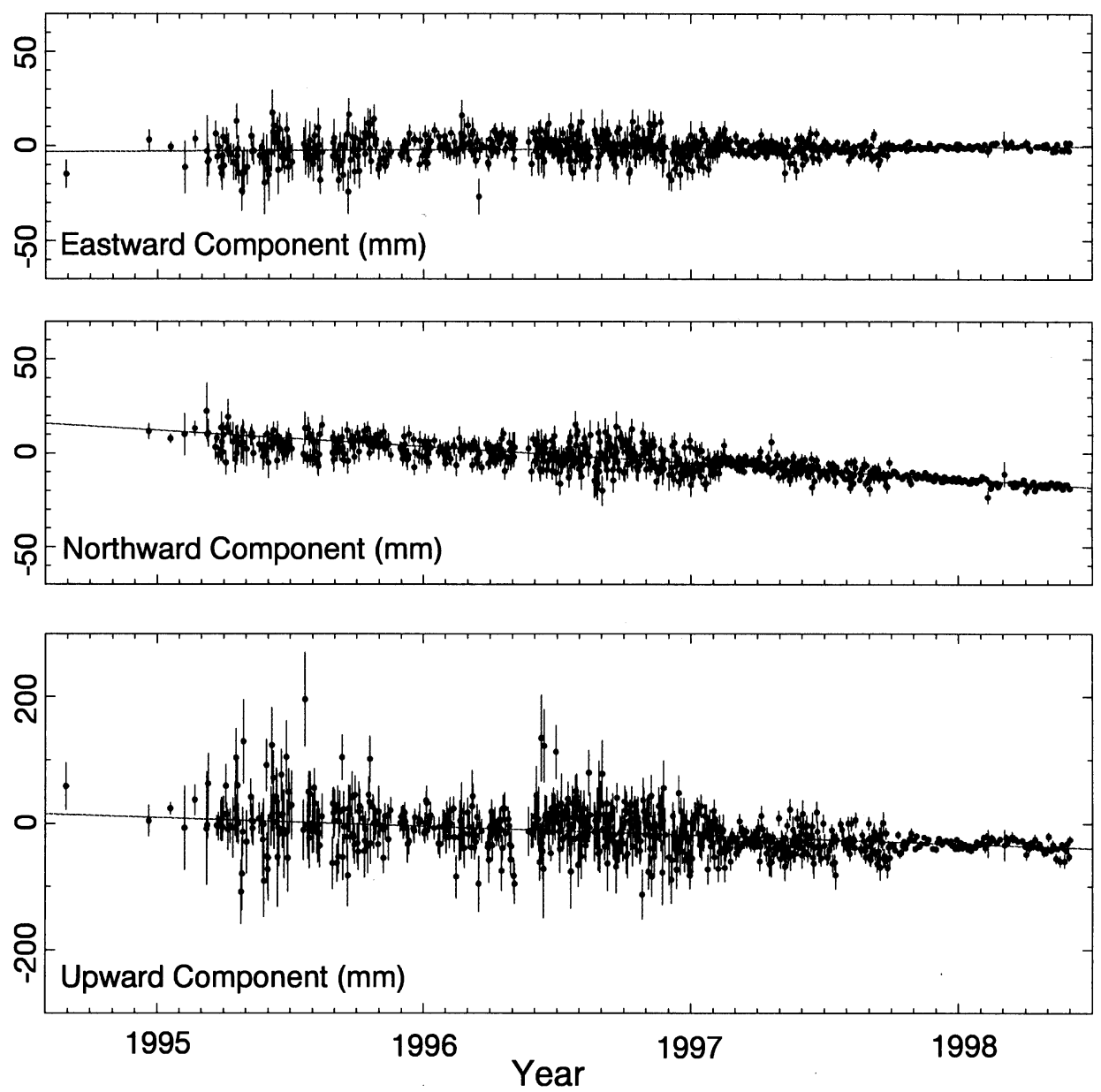

Fig. 10. Site position of the Koganei station in the local cartesian coordinate system. Estimated uncertainties in the estimates are shown by the one-sigma formal error bars. A straight line in each figure is showing the best-fit line to the estimated site position. 
Table 5. Baseline lengths $L_{0}$ on the epoch of September 1, 1996 and their rates of change estimated for six baselines by the least squares estimation.

\begin{tabular}{lcc}
\hline Baseline & $\begin{array}{c}\text { Baseline Length } L_{0} \\
\text { Sep. 1, 1996 }(\mathrm{mm})\end{array}$ & $\begin{array}{c}\text { Rates of Change } \\
(\mathrm{mm} / \text { year })\end{array}$ \\
\hline Koganei-Kashima & $109099658.35 \pm 0.15$ & $-1.54 \pm 0.16$ \\
Koganei-Miura & $57734572.59 \pm 0.23$ & $-11.88 \pm 0.25$ \\
Koganei-Tateyama & $91871045.70 \pm 0.30$ & $-14.04 \pm 0.32$ \\
Kashima-Miura & $123379071.57 \pm 0.25$ & $-3.87 \pm 0.28$ \\
Kashima-Tateyama & $134771056.84 \pm 0.39$ & $-8.42 \pm 0.42$ \\
Miura-Tateyama & $35028135.93 \pm 0.30$ & $-2.27 \pm 0.32$ \\
\hline
\end{tabular}

sitions for the same period yielded the weighted rms residual as about $2 \mathrm{~mm}$ for the horizontal components and $9 \mathrm{~mm}$ for the vertical component. These values can be considered as the current accuracy of the VLBI measurements with the Key Stone Project VLBI network.

Table 5 tabulates the lengths of six baselines estimated for the epoch of September 1, 1996, and their rates of change estimated from all the data by least squares estimation. Both the baseline lengths and their rates of change have been precisely determined from more than three years of experiments. These results, as well as the three-dimensional station positions, are updated every time either an initial data analysis is done or a reanalysis is done using updated Earth Rotation Parameters, and they are made publicly available from the Internet via WWW (http://ksp.crl.go.jp/) and ftp (ksp.crl.go.jp). The correlated amplitudes of observed radio sources are also accessible through the Internet and can be utilized for astronomical purposes.

\section{Concluding Remarks and Future Improve- ments}

Both the VLBI observation control system and the VLBI data analysis system for the Key Stone Project have been designed for automation and robustness through the use of redundancy. The unique features of the Key Stone Project VLBI system have resulted in regular geodetic VLBI experiments with four VLBI stations with a time interval of one or two days. Results from the experiments are produced quickly after the experiment. In the tape-based VLBI mode, the results can be obtained within two days from the last observation in the experiment, whereas the time required between the last observation and the data analysis was reduced to a few minutes in the real-time VLBI mode. From 23.5 hours of experiments, the horizontal and vertical position uncertainties were about 2 and $9 \mathrm{~mm}$, respectively, in a sense of internal estimation error evaluated by one sigma standard deviation.

To improve the estimation accuracy, the key is to increase the number of successful observations as well as to improve the theoretical models. The number of observations in an experiment can be increased by reducing the observation time for each radio source. Data synchronization can be performed while the antenna is slewing to change from the previous radio source to the next one. The number of obser- vations in an experiment might also be increased significantly if the syncronization time in the cross correlation processing is shortened by a fine tuning of the data syncronization algorithm. The observation time can be shortened for strong radio sources if the minimum integration time is decreased from the present value of 50 seconds. If the minimum integration time is set too short, however, interferometric fringes may not rotate enough and the obtained delays may have errors that cannot be ignored. Therefore, the minimum integration time should be carefully investigated. Although only the group-delay data are used at present, the usage of the phase-delay data have a potential to improve the precision of the VLBI measurements (Herring and Pearlman, 1993). The possibility of the phase delay measurements will be investigated in the near future by using the Key Stone Project VLBI network.

The availability of the VLBI data obtained from the regular Key Stone Project experiments will be greatly improved if the results are generated in SINEX (Solution Independent Exchange) format, which will enable these results to be combined with other geodetic measurements including the GPS and SLR. A detailed comparison of the VLBI results with results from GPS and SLR measurements is one of the major purposes of the Key Stone Project. The implementation of the automated SLR and GPS data analysis system is on the way and soon the comparison will become possible.

Acknowledgments. The authors would like to thank all the members of the Communications Research Laboratory who contributed to the development of the Key Stone Project VLBI system. The high-speed ATM network system for the real-time VLBI observations in the Key Stone Project was developed under the collaboration with NTT Optical Network System Laboratory. Some of the software routines have been developed by NASA Goddard Space Flight Center and by Dr. Thomas Herring of Massachusetts Institute of Technology.

\section{References}

Anderson, A. J. and A. Cazenave (Eds.), Space Geodesy and Geodynamics, 490 pp., Academic Press, London, 1986.

Clark, T. A., B. E. Corey, J. L. Davis, G. Elgered, T. A. Herring, H. F. Hinteregger, C. A. Knight, J. I. Levine, G. Lundqvist, C. Ma, E. F. Nesman, R. B. Phillips, A. E. E. Rogers, B. O. Rönnäng, J. W. Ryan, B. R. Schupler, D. B. Shaffer, I. I. Shapiro, N. R. Vandenberg, J. C. Webber, and A. R. Whitney, Precision geodesy using the Mark-III Very Long Baseline Interferometer system, IEEE Trans. Geoscience and Remote Sensing, GE-23, 438-449, 1985.

Eubanks, T. M., Variations in the orientation of the Earth, in Contributions of 
Space Geodesy to Geodynamics: Earth Dynamics, edited by D. E. Smith and D. L. Turcotte, pp. 1-54, American Geophysical Union, Washington D.C., 1993.

Herring, T. A. and M. R. Pearlman, Future developments and synergism of space geodetic measurement techniques, in Contributions of Space Geodesy to Geodynamics: Technology, edited by D. E. Smith and D. L. Turcotte, pp. 21-25, American Geophysical Union, Washington D.C., 1993.

Kiuchi, H., S. Hama, and M. Imae, K-4 and KSP VLBI terminal, in Proceedings of the Technical Workshop for APT and APSG 1996, pp. 166-170, 1996a.

Kiuchi, H., M. Imae, T. Kondo, and M. Sekido, Real-time VLBI of the KSP, in Proceedings of the Technical Workshop for APT and APSG 1996, pp. 125-129, 1996b.

Kondo, T., H. Kiuchi, and M. Sekido, KSP correlator and data reduction system, in Proceedings of the Technical Workshop for APT and APSG 1996, pp. 188-191, 1996.

Kondo, T., N. Kurihara, Y. Koyama, M. Sekido, R. Ichikawa, T. Yoshino, J. Amagai, K. Sebata, M. Furuya, Y. Takahashi, H. Kiuchi, and A. Kaneko, Evaluation of repeatability of baseline lengths in the VLBI network around Tokyo metropolitan area, Geophys. Res. Lett., 25, 1047-1050, 1998.

Koyama, Y., Excess westward velocities of Minamitorishima (Marcus) and Kwajalein VLBI stations from the expected velocities based on rigid motion of the Pacific Plate, J. Geodetic Soc. Jpn., 42, 43-56, 1996.

Koyama, Y., K. Heki, M. Imae, T. Kondo, H. Kuroiwa, Y. Sugimoto, F. Takahashi, T. Yoshino, C. Miki, and J. Amagai, Horizontal movements of Marcus VLBI station due to the Pacific Plate motion, Proc. CRCM'93, special issue of J. Geodetic Soc. Jpn., 117-122, 1994.

Kunimori, H., F. Takahashi, M. Imae, Y. Sugimoto, T. Yoshino, T. Kondo, K. Heki, S. Hama, Y. Takahashi, H. Takaba, H. Kiuchi, J. Amagai, N. Kurihara, H. Kuroiwa, A. Kaneko, Y. Koyama, and K. Yoshimura, Contributions and activities of Communications Research Laboratory under the cooperation with Crustal Dynamics Project, in Contributions of Space Geodesy to Geodynamics: Technology, edited by D. E. Smith and D. L. Turcotte, American Geophysical Union Geodynamics Series, 25, pp. 65 79, 1993.

Kurihara, N., K. Uchida, F. Takahashi, M. Imae, T. Yoshino, S. Hama Y. Takahashi, and Key Stone Project Group, The crustal deformation monitoring system for the Tokyo Metropolitan Area (Key Stone Project), in Proceedings of the Technical Workshop for APT and APSG 1996, pp. 65-69, 1996.

Ryan, J. W., T. A. Clark, C. Ma, D. Gordon, D. S. Caprette, and W. E. Himwich, Global scale tectonic motions measured with CDP VLBI data, in Contributions of Space Geodesy to Geodynamics: Crustal Dynamics, edited by D. E. Smith and D. L. Turcotte, pp. 37-49, American Geophysical Union, Washington D.C., 1993.

Steufmehl, H., AUTOSKED-Automatic creation of optimized VLBI observing schedules, in Proceedings of the 8th Working Meeting on European VLBIfor Geodesy and Astrometry, Report MDTNO-R-9243, Survey Dep of Rijkswaterstat Delft, IV22-IV29, 1983.

Y. Koyama (e-mail: koyama@crl.go.jp), N. Kurihara, T. Kondo, M. Sekido, Y. Takahashi, H. Kiuchi, and K. Hek 